\title{
Mehr Zieloffenheit in der Suchttherapie
}

\section{Urs Thalmann}

Soziologe, lic. phil., Zentrumsleiter Mühlhof - Zentrum für Suchttherapie und Rehabilitation, Tübach SG

Abstinenz gilt oft als einziger Weg aus der Alkoholsucht. Zieloffene Therapien, die auch eine Reduktion des problematischen Konsums als Veränderungsoption integrieren, werden zwischenzeitlich als vielversprechende Ergänzung gewürdigt, um die Behandlungs- und Erfolgsquote zu steigern. Dies erfordert eine individuelle Behandlung der Patienten und eine Abkehr von dogmatischen Therapieansätzen. Diese Position vertraten auch Suchtspezialisten an einer Fachtagung in St. Gallen.

Die Mehrheit der Alkoholtherapien verfolgt eine abstinenzorientierte Zielsetzung. Der absolute Alkoholverzicht wird meistens als einzige Behandlungsoption angepriesen, obwohl dieses Ziel für Betroffene oft sehr hoch, für manche fast unerreichbar erscheint.

Doch bereits heute überwindet die Mehrheit der Alkoholabhängigen ihre Sucht durch eine Reduktion ihres Trinkverhaltens und nicht durch einen völligen Verzicht. Suchttherapie kann letztlich nur erfolgreich sein, wenn ein Patient* von den Zielen seiner Behandlung überzeugt ist und von sich aus eine Veränderung will. Rückfälle aus der Abstinenz sind denn auch die Regel und nicht die Ausnahme.

\section{Individuelle Zielsetzungen}

Ohne die Abstinenz als Königsweg in Frage zu stellen, fordern Experten wie Prof. Dr. Joachim Körkel deshalb einen offeneren Umgang mit den Zielsetzungen von Alkoholkranken. Für Personen, bei denen eine Abstinenz nicht in Frage kommt, sollen alternative Therapieangebote geschaffen werden. Eine Reduktion sei allemal besser, als die Betroffenen ihrem Schicksal zu überlassen. Dies betrifft beispielsweise Rückfallpatienten oder Menschen, die sich einer Therapie verschliessen, weil sie der Überzeugung sind, nie gänzlich auf Alkohol verzichten zu können. Ärzte und Therapeuten

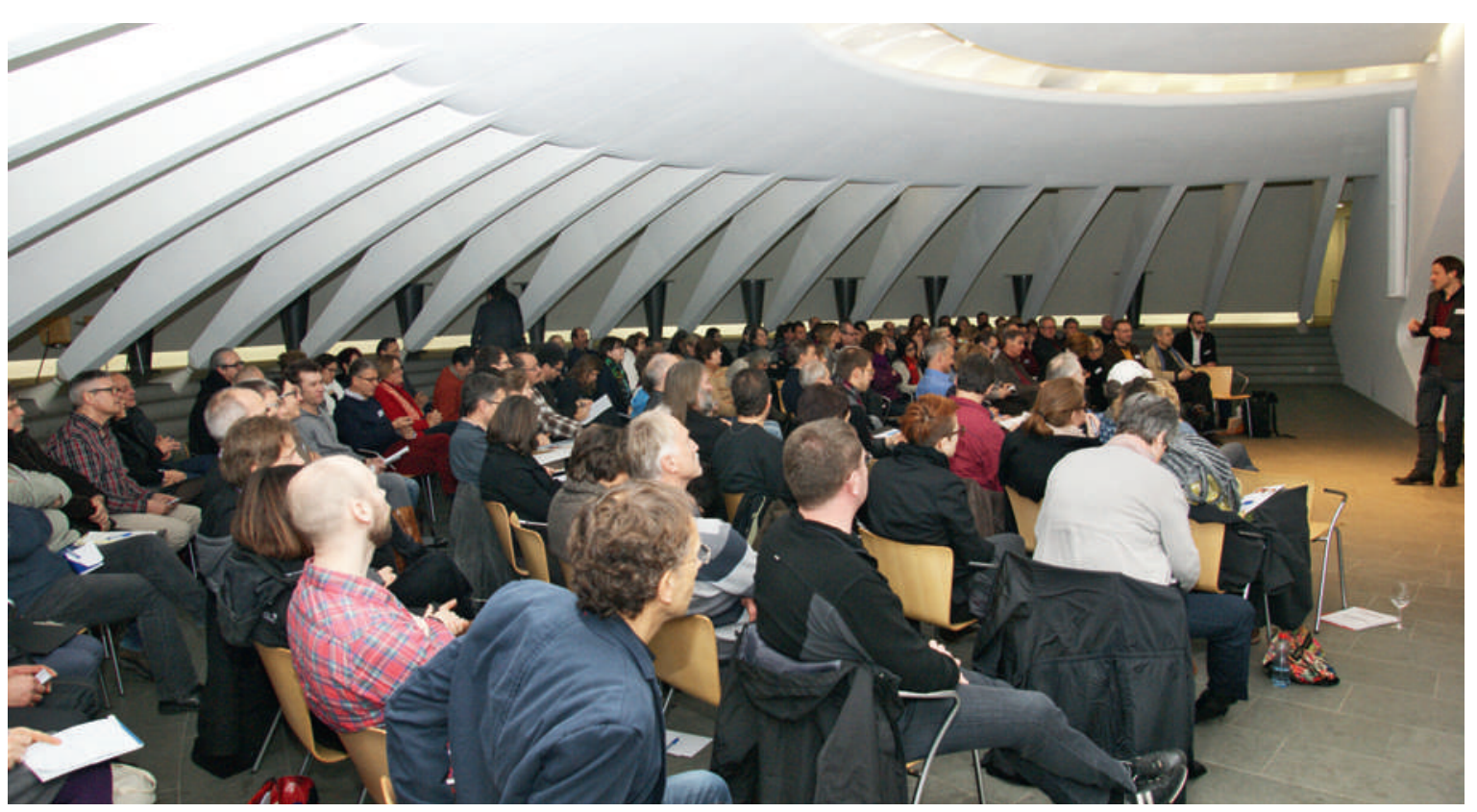

Bei der vielbeachteten Tagung der Stiftung Mühlhof im St. Galler Pfalzkeller sprachen prominente Vertreter zieloffener Therapien als Ausweg aus der Alkoholsucht. 
sollen deshalb davon abrücken, bereits im Vornherein zu wissen, was das Beste für die jeweiligen Patienten ist. Bei einer individuellen Behandlung liegt es beim Patienten, sich seine Ziele selbst zu setzen und zu bestimmen, wie der problematische Konsum reduziert werden kann. Mit Hilfe eines Plans wird festgehalten, wann, wie viel und in welchem Rahmen Alkohol konsumiert werden darf. Die Kontrolle erfolgt über ein Tagebuch, das der Patient selbst führt.

\section{Erhöhung der Behandlungsquote}

Gemäss Expertenschätzungen lassen heute 90-95\% der Alkoholabhängigen ihre Sucht nicht behandeln. Im Wissen um diese Problematik bietet die zieloffene Suchtarbeit zahlreiche Vorteile. So sind die Eintrittshürden mit einer individuellen Zielsetzung wesentlich tiefer, als wenn vom Therapeuten von Beginn an die Maximalforderung "Abstinenz" aufgestellt wird. Auf diese Weise lässt sich die Behandlungsquote von Alkoholkranken markant erhöhen. Auch Personen, die bei einer abstinenzorientierten Therapie rückfällig geworden sind, lassen sich durch eine Erweiterung der Behandlungsmöglichkeiten besser motivieren, einen neuen Anlauf zu nehmen. Der Übergang zur «Abstinenz» ist durch eine Therapie, die sich an einer Konsumreduktion orientiert, nicht ausgeschlossen. Die zieloffene Behandlung kann im Idealfall einen graduellen Übergang zur Abstinenz darstellen. Gemäss dem Winterthurer Suchtmediziner Dr. med. Toni Bertel stellt sich die Grundsatzfrage «Abstinenz» oder «kontrollierter Konsum» nicht. Primär gehe es darum, dass der Patient eine methodisch individuelle und erfolgsversprechende Unterstützung erhält, die im richtigen Zeitpunkt greift und vom zu Behandelnden angenommen wird. Internationale Studien belegen den Erfolg der zieloffenen Alkoholsuchttherapie. Die durchschnitt-

\section{Résumé: Plus d'ouverture dans le traitement des addictions}

L'abstinence est souvent présentée comme le seul moyen de traiter la dépendance à l'alcool. Des experts de l'addiction appellent cependant à davantage d'ouverture dans la définition des objectifs de traitement, afin $d^{\prime}$ 'inciter le patient à modifier son comportement. Les personnes concernées devraient ainsi pouvoir fixer individuellement les objectifs de leur thérapie et vérifier elles-mêmes leurs progrès. Réduire les contraintes liées à une thérapie permet non seulement d'augmenter le nombre de personnes traitées mais également d'améliorer les chances de succès. Par ailleurs, les personnes qui ont expérimenté une rechute seraient plus motivées à reprendre leur thérapie si les objectifs leur paraissent plus réalistes que l'abstinence. Pour les thérapeutes et les institutions dans le domaine de l'addiction, ce changement de paradigme suppose de renoncer à des objectifs standards pour développer des approches thérapeutiques individuelles.

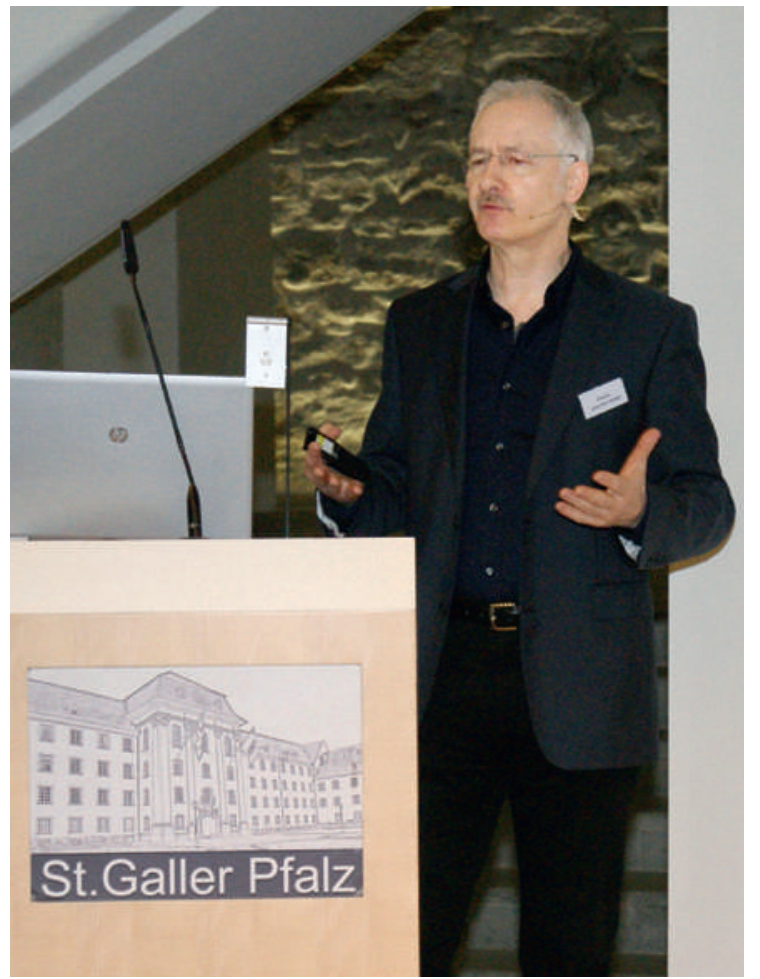

Der Nürnberger Suchtforscher Professor Joachim Körkel fordert einen offeneren Umgang mit Alkoholkranken.

liche Erfolgsquote liegt bei 65\%, wobei der Alkoholkonsum im Mittel um 50\% reduziert wird. 10-30\% der behandelten Patienten gelingt schliesslich sogar der Schritt in die Abstinenz [1].

\section{Gleichzeitige Behandlung von Begleit- erkrankungen}

Bei Suchterkrankungen gilt es, grundsätzlich früh, rasch und wiederholt zu intervenieren. Nur so können physische Schäden vermindert werden. Substanzstörungen sind jedoch äusserst komplex und basieren auf einer Vielzahl möglicher Ursachen. Oft gehen sie auch mit körperlichen, psychischen und sozialen Problemen einher. Die Erfahrung zeigt, dass bessere Erfolge erzielt werden, wenn Begleiterkrankungen parallel zur Sucht behandelt werden.

\section{Konsequenzen für stationäre Sucht- therapie}

Bis anhin sind die Behandlungsangebote für Alkoholkranke in den meisten stationären Therapiezentren und Kliniken lediglich auf Abstinenz ausgerichtet. Für eine Implementation der zieloffenen Suchtarbeit ist daher ein Umdenken auf Stufe der Therapeuten sowie eine Anpassung der entsprechenden Rahmenbedingungen nötig. Dabei ist aber zu beachten, dass Patien- 


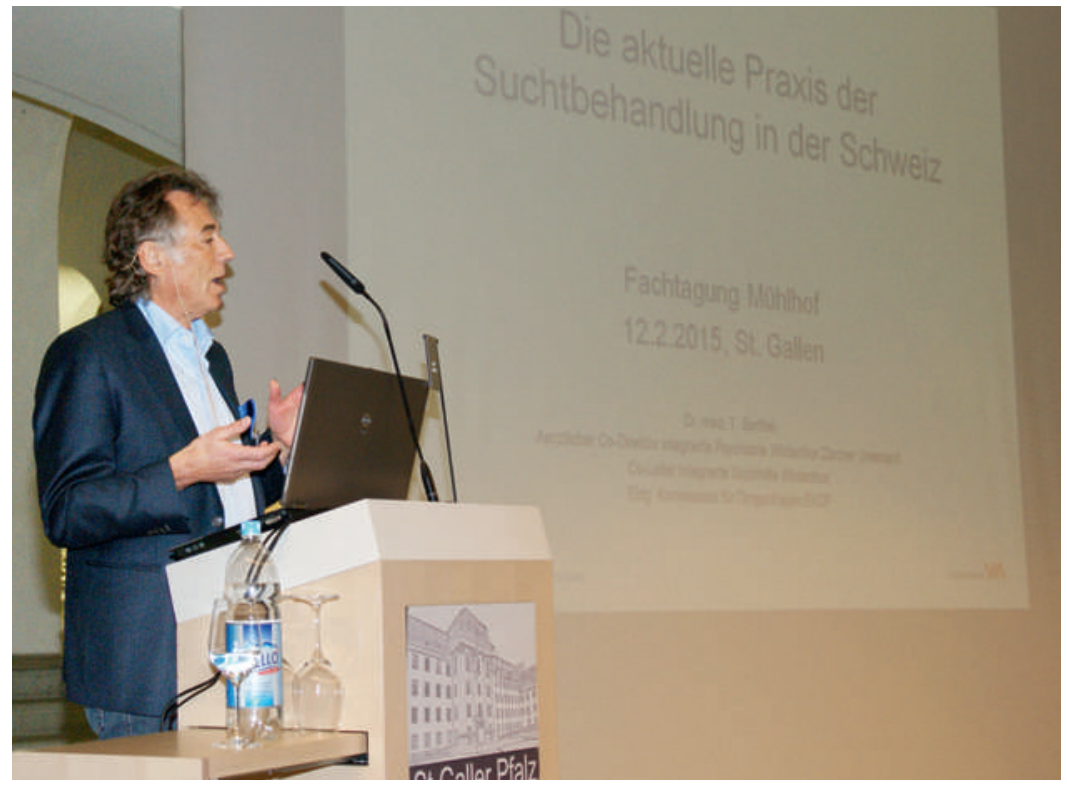

Auch der Co-Direktor der Integrierten Psychiatrie Winterthur und Präsident der Eidgenössischen Kommission für Suchtfragen Toni Berthel vertritt die sog. "Zieloffenheit", eine Öffnung von Suchttherapien für individuelle Lösungen.

ten mit dem Ziel der Abstinenz in ihrem Vorhaben nicht gestört werden. So ist davon auszugehen, dass im Rahmen von stationären Behandlungen der Konsum von Alkohol weiterhin sehr restriktiv gehandhabt werden muss. Ähnliche Spannungsfelder betreffen den Konsum im Ausgang, Regelverstösse und deren therapeutischer Umgang. Es muss geklärt werden, wie weit die Selbstkontrolle des Patienten reicht und ab wann Therapeuten einschreiten sollen und dürfen.

\section{Humanistisches Menschenbild}

Im Zentrum der zieloffenen Suchttherapie steht ein humanistisches Menschenbild. Die Würde der Patienten erfordert demnach, dass keine Behandlung gegen

\section{Die Eintrittshürden in eine Therapie sind mit einer individuellen Zielsetzung wesentlich tiefer.}

deren Willen vorgenommen wird und diese sich mit den Zielen und Massnahmen einverstanden erklären müssen. Patienten sollen trotz des Kontrollverlusts, den sie durch die Sucht erlitten haben, als grundsätzlich selbstbestimmende Individuen behandelt werden. Für dogmatische und fundamentalistische Ansätze gibt es keinen Raum. Jede Alkoholsucht hat individu- elle Ursprünge und Ausprägungen. Jeder Betroffene hat damit auch den Anspruch, individuell behandelt zu werden. Nebst der Abstinenz sollte es somit auch Platz für alternative Therapieziele geben.

\section{Zusammenfassung}

Abstinenz wird hierzulande oft als einzige Möglichkeit dargestellt, die Alkoholsucht zu überwinden. Suchtexperten fordern dazu auf, zieloffene Therapien zu ermöglichen, die auf eine Änderung des problematischen Konsumverhaltens abzielen. Betroffene sollen die Zielsetzungen ihrer Therapie individuell festlegen können und die Einhaltung der getroffenen Massnahmen selbst überprüfen. Durch niedrigere Eintrittshürden können nicht nur die Behandlungs-, sondern auch die Erfolgsquoten der Therapien gesteigert werden. Zudem können rückfällig gewordene Personen besser motiviert werden, sich erneut einer Therapie zu stellen, wenn erreichbare Zielsetzungen an die Stelle der Abstinenz treten. Für Therapeuten und die Institutionen im Bereich der Suchttherapie erfordert dies eine Abkehr von vorgefertigten Zielsetzungen und die Weiterentwicklung bisheriger Behandlungsansätze hin zu individueller Suchttherapie.

\section{Literatur}

1 Körkel J. Alkoholtherapie: Vom starren Abstinenzdogma zu einer patientengerechten Zielbestimmung. Suchtmed. 2014; 16(5):211-2.

\section{Nützliche Links}

(Verlinkung in SÄZ-Online-Ausgabe)

- Meyer T. Kontrolliertes Trinken. Stand der Debatte in der Schweiz. www.yumpu.com/de/document/view/6479992/kontrolliertestrinken-stand-der-debatte-in-der-sucht-schweiz

- Körkel J. Zwischen freiem Willen und biologischem Determinismus. www.kontrolliertes-trinken.de/kontrolliertes-trinken/ de/3/3/hintergrund/determinismus.aspx

- Körkel J. Kontrolliertes Trinken / Begründung und Wirksamkeit. www.kontrolliertes-trinken.de/kontrolliertes-trinken/de/3/2/ hintergrund/wirksamkeit.aspx

- Berthel T, Gallego S. Mässigung: Das elfte Gebot. www.ipw.zh.ch/ internet/gesundheitsdirektion/ipw/de/aktuell/ mitteilungen/2014/rausch/ jcr content/contentPar/ downloadlist_1413201185645/downloaditems/rausch_und konsumkom.spooler.download.1413200096032.pdf/ SuchtMagazin 42014 Berthel Gallego.pdf

- Klingemann H, Aeberhard M: Biographie und Suchtkarrieren 1988-2002. www.researchgate.net/publication/237228655 Biographie_und_Suchtkarrieren_1988_-2002_Longitudinale Fallanalysen_von_Alkohol-_und_Heroinselbstheilerinnen

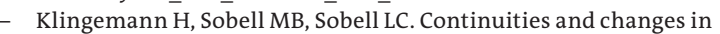
self-change research. www.readcube.com/ articles/10.1111\%2Fj.1360-0443.2009.02770.x?r3 referer=wol\&tracking action=preview click\&show checkout=1\&purchase_referrer=onlinelibrary.wiley. com\&purchase_site_license=LICENSE_DENIED_NO_CUSTOMER 